\title{
A Novel Polyclonal Rabbit Immunoglobulin G Crystallisation Approach Using 3D Nanotemplate
}

\author{
Kuan L. Tan, Peck L. Kiew, Nilanjon Naskar, and Jerry Y. Y. Heng
}

\begin{abstract}
Protein crystallisation is the formation process of protein crystal from a supersaturated solution in which metastable supersaturated solution containing the protein interested reaching a stable low energy state by reduction of solute concentration. In recent years, protein crystallisation has spurred the interest among researchers to explore its potential application as one of the downstream processing methods for low-cost production and purification of proteins. Heterogeneous template nucleant is the key factor for successful protein crystallisation. It reduces the energy barrier for nucleation by constrains the protein molecules and promotes the protein molecules to aggregate in crystalline order in order to facilitate the protein crystallisation. This report focuses on the identification of optimal crystallising conditions for polyclonal immunoglobulin G subclass 4 (IgG4) proteins by employing three-dimensional (3D) nanotemplates as the facilitating nucleant. The comparison of crystal growth of the polyclonal IgG4 was performed with and without the presence of 3D nanotemplate. The results revealed that mesoporous 3D nanotemplates were able to facilitate crystallisation process of polyclonal IgG4. The optimal crystallisation conditions for polyclonal IgG4 was successfully determined. Polyclonal IgG4 was found to crystallise optimally at a protein concentration of $10 \mathrm{mg} / \mathrm{ml}$ using 3D nanotemplates with pore size $10 \mathrm{~nm}$, PEG 8000 as precipitant, Calcium Acetate and Imidazole as buffer at the incubation temperature and $\mathrm{pH}$ of $20^{\circ} \mathrm{C}$ and 7 respectively.
\end{abstract}

Index Terms-3D nanotemplate, crystallisation, IgG4, heterogeneous nucleant.

\section{INTRODUCTION}

Protein crystallisation was developed in the second half of 19th century and it has been the research subject of significant interest for more than 35 years. However, crystallisation of protein is still based on empirical approaches until now. The fact that protein crystallisation was mainly attempted by trial and error instead of systematic understanding of the crystallisation process of proteins has driven the researchers to focus on the investigation of the crystallisation approaches and the understanding of the mechanism behind it. The motivation to crystallise proteins is also driven by the need to undertake the X-ray structural determinations of proteins and the exploration of the potential use of the protein crystallisation as a low cost downstream processing method [1]. On top of that, protein crystallisation has also been a

Manuscript received March 10, 2016; revised August 15, 2016.

Kuan L. Tan and Peck L. Kiew are with UCSI University, Malaysia (e-mail: leonttkl@hotmail.com, kiewpl@ucsiuniversity.edu.my).

Nilanjon Naskar is with Indian Institute of Technology Kharagpur, India (e-mail: cnilanjon.naskar15@imperial.ac.uk).

Jerry Y. Y. Heng is with Imperial College London, UK (e-mail: jerry.heng@imperial.ac.uk). popular topic for many other areas of science particularly in nutraceuticals, environmental sciences, food products, semiconductors, non-linear optics, and synthetic systems [2]-[8].

According to Diao et al. [9], the important role of surface chemistry in heterogeneous nucleation of protein have been clearly demonstrated and studied, but there is limited understanding and investigation on the effect of various surface chemistries to the protein nucleation outcomes. Therefore, this research aims to investigate the effect of an engineered nucleating surface, namely three-dimensional (3D) nanotemplate to the protein crystallisation. Using 3D nanotemplates as the heterogeneous nucleants, the optimum crystallisation conditions of an industrially relevant antibody - polyclonal immunoglobulin $\mathrm{G}$ subclass 4 ( $\mathrm{IgG} 4$ ) will be determined. The author hypothesised that the application of the 3D nanotemplates will facilitate the protein crystallisation process and significantly reduce the induction time of crystallisation. To investigate the effectiveness of the 3D nanotemplates in facilitating and inducing protein nucleation, nucleation induction time statistics will be utilised for the comparison of the crystal growth with and without the application of 3D nanotemplates.

The outcome of the research is anticipated to assist in the exploration of potential applications of controlled protein crystallisation using 3D nanotemplates as the alternative downstream processing method for the biopharmaceutical manufacturing and other possible fields. The application of protein crystallisation as a purification and separation method of proteins will reduce the separation and purification cost of proteins in the downstream processing. Apart from that, protein crystallisation facilitated by 3D nanotemplate are expected to produce large and high diffraction quality protein crystals that are suitable for the structural determination purposes. The accurate structural determination of proteins are essential for the study of protein crystallisation mechanism and it will help to unveil the functionalities and behaviours of many proteins which are still not being crystallised.

Heterogeneous nucleation is a crystallisation process in which the crystallisation system contains foreign surfaces such as dust particles, glass walls, etc. Due to the presence of these foreign surfaces, the aggregation and ordering of protein molecules occur at the nucleation sites on these foreign particles [10]-[12]. This reduces the energy barrier or nucleation barrier for new phase formation and porous surfaces can locally confine clusters of protein molecules dispersed in the supersaturated solution in order to promote aggregation of protein molecules [12]. Therefore, 
heterogeneous nucleants are well-known to reduce the change in free energy required for nucleation induction and hence protein crystals can be obtained at low solute concentration.

Using classical nucleation theory, heterogeneous nucleation can be attained at a much faster rate as compared to homogeneous nucleation which is defined as the spontaneous nucleation without preferential nucleation sites [13]. In practical, heterogeneous nucleation is more commonly employed compared to the latter. Despite the fact that protein nucleation is inherently a kinetic process, the investigation of the role of nucleating surfaces in crystallisation is still very limited from the perspective of nucleation kinetics [9]. Therefore, investigating the template assisted nucleation or simply heterogeneous nucleation with the well-defined template will help to understand the nucleation mechanisms and nucleation kinetics [13]. The interest of this research is on the comparison of nucleation rates between the heterogeneous nucleation on engineered surfaces (3D nanotemplate) with homogeneous nucleation. 3D nanotemplates are highly porous engineered surfaces which are believed to facilitate the nucleation of protein and reduce the nucleation induction time.

In this work, protein crystallisation was attempted by employing the concept of heterogeneous nucleation suggested by Shah et al. [14] and the optimum crystallisation conditions for crystallisation of an entire full length intact polyclonal rabbit IgG4 using mesoporous 3D nanotemplates were investigated. 3D nanotemplates are the engineered mesoporous silica with highly-ordered and narrow distributions of nano-confined cavities which are believed to facilitate the nucleation of protein and reduce the nucleation induction time significantly. The objectives of this work are (1) To identify the optimum crystallisation conditions of polyclonal IgG4; (2) To compare the crystals growth with and without the presence of 3D nanotemplate; (3) To conduct induction time analysis for polyclonal IgG4 crystallisation for nucleation rate evaluation. Subsequently, the induction time statistics was presented for the polyclonal IgG4 crystallisation.

\section{EXPERIMENTAL METHODS}

\section{A. Materials}

Rabbit serum derived polyclonal IgG4 antibody was purchased from Sigma-Aldrich, Dorset, United Kingdom and was used without further purification. Calcium acetate hydrate, imidazole, sodium hydroxide, acetic acid and PEG 8000 were purchased from Sigma-Aldrich also.

\section{B. Preparation of Buffer and Precipitant Solution}

The buffer solution was prepared by mixing $0.1 \mathrm{M}$ imidazole with $0.2 \mathrm{M}$ calcium acetate whereas PEG 8000 was used as the precipitant for polyclonal IgG4 crystallisation.

\section{Hanging Drop Crystallisation of Proteins}

48-well clear polystyrene microplates were used for crystallisation experiments. Buffer and precipitant solution were sterile-filtered using 0.22 um filter unit. The $\mathrm{pH}$ was adjusted by adding a solution of $1 \mathrm{M}$ acetic acid or $1 \mathrm{M}$ sodium hydroxide when required. High vacuum grease was applied along the top edge of the raised reservoir of the microplate to ensure the reservoir wells are airtight. Each reservoir well was filled with $750 \mu \mathrm{l}$ of precipitant solution. Droplets of $6 \mu \mathrm{l}$ volumes of protein solution were created on the $22 \mathrm{~mm}$ rectangular glass cover-slip and the precipitant solution of the equal volume was added to the droplet. Edges of the coverslips were carefully inverted and placed on top of the reservoir, pressed gently to relieve pressure and twisted carefully to ensure proper seal. These crystallisation cells were then carefully placed in the temperature-controlled incubator (Surface Measurements Systems, UK) at $20{ }^{\circ} \mathrm{C}$ and the crystallisation behaviour was monitored.

\section{Observation of Crystallisation}

Olympus BX51 M reflective microscope (Olympus UK Ltd., UK), which is equipped with polarising filters and Olympus DP70 digital camera (Olympus UK Ltd., UK) for digital imaging were used for the qualitative observation of crystallisation process. The crystal length was determined from the microphotographs using the Olympus Image Analysis Software. Polyclonal IgG4 crystals and opaque 3D nanotemplates were distinguished through crystal transparency and surface structure differences between them.

\section{RESULTS AND DISCUSSION}

\section{A. Crystallisation of Polyclonal IgG4 on 3D Nanotemplates}
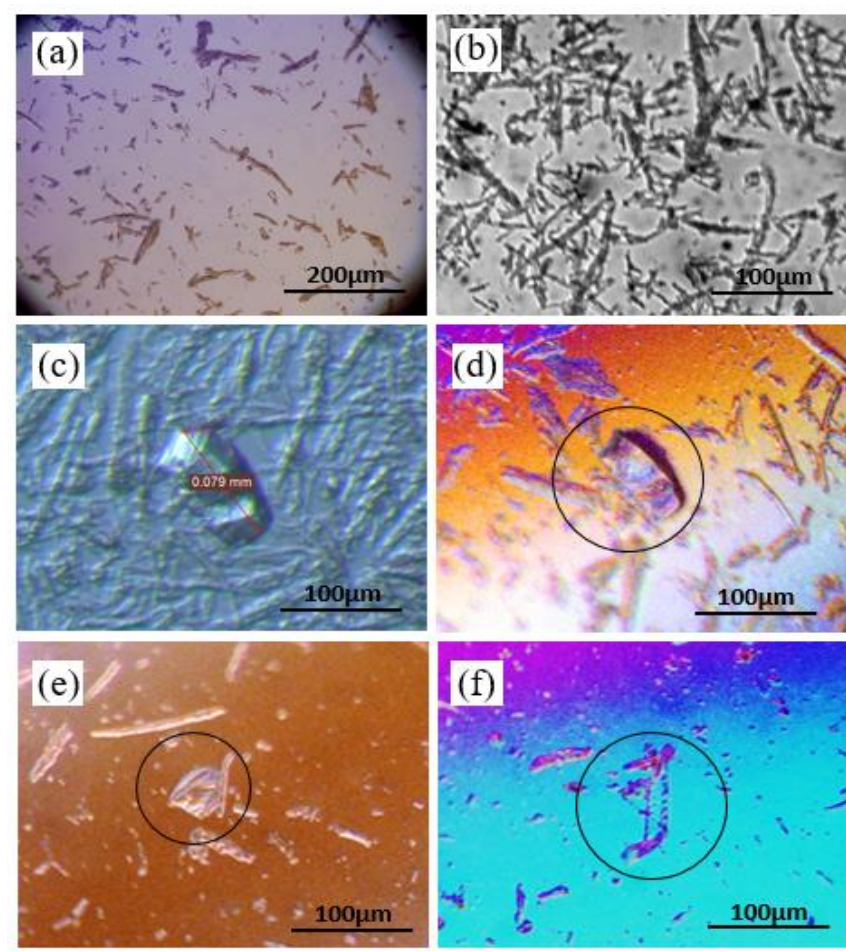

Fig. 1. Microphotographs of 3D nanotemplates and polyclonal IgG4 crystals (a) 3D nanotemplates, scale bar $200 \mu \mathrm{m}$ (b) 3D nanotemplates, scale bar $100 \mu \mathrm{m}$ (c), (d), (e), and (e) IgG4 crystals crystallised on the 3D nanotemplates.

Polyclonal IgG4 antibody was crystallised successfully on the $3 \mathrm{D}$ nanotemplates with pore size of $10 \mathrm{~nm}$. The incubation temperature and $\mathrm{pH}$ were set at $20^{\circ} \mathrm{C}$ and 7 respectively. The 
protein concentration used was $10 \mathrm{mg} / \mathrm{ml}$. From Fig. 1, it can be observed that well-faceted polyclonal IgG4 crystals were formed on the surfaces of 3D nanotemplate. The opaque nature and rough surface of the $3 \mathrm{D}$ nanotemplate clearly distinguish the $\mathrm{IgG} 4$ crystals from it. Formation of the crystals and the time required for them to be visible took approximately 6 hours. The microphotographs were taken at $10 \mathrm{x}$ magnification. The author proposes that the success in IgG4 crystallisation is due to the fact that optimum crystallisation condition had been achieved by employing 3D nanotemplates as the nucleant. As proposed by Shah et al. [9], 3D nanotemplate stabilises protein nuclei within the mesopores by local immobilisation. The IgG4 proteins diffuse into the mesopores and the local immobilisation effect will lower the free energy barrier by promoting the formation of nuclei. The results here supported their study.

\section{B. Crystallisation of Polyclonal IgG4 in Absence of $3 D$ Nanotemplate}

The possibility of IgG4 crystallisation in the same condition without employing 3D nanotemplate was also investigated. It was found that even after two weeks, formation of the crystals was not observed. Therefore, it can be concluded that Crystallisation of IgG4 is not possible without the presence of 3D nanotemplate.

\section{Crystallisation of Polyclonal IgG4 at Varying Protein Concentration}

The IgG4 concentration was varied from 2 to $10 \mathrm{mg} / \mathrm{ml}$ whereas the percentage of PEG was kept constant. It was found that $10 \mathrm{mg} / \mathrm{ml}$ was the optimal protein concentration for the crystallisation process. At other protein concentrations other than $10 \mathrm{mg} / \mathrm{ml}$, no IgG4 protein is crystallised. At protein concentrations higher than $10 \mathrm{mg} / \mathrm{ml}$, precipitates were formed immediately without forming any IgG4 crystals. In the other hand, at lower protein concentrations (less than $10 \mathrm{mg} / \mathrm{ml}$ ), no IgG4 crystals formed as the protein concentrations are below the solubility of IgG4 protein.

\section{Induction Time Statistics}

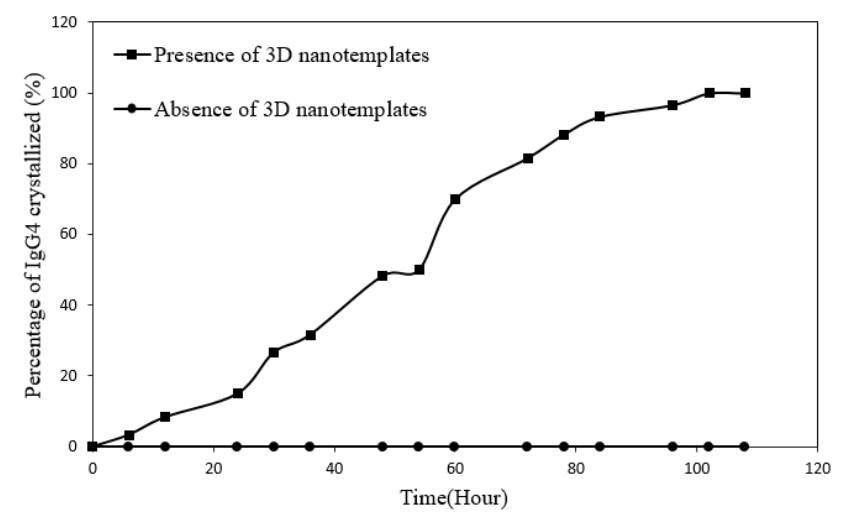

Fig. 2. Cumulative probability distribution of induction time for polyclonal IgG4 crystallisation with and without the presence of 3D nanotemplate.

Induction time analysis could be employed to correlate the nucleation theory with the experimental crystallisation in this research. Induction time statistics was generated based on the cumulative probability distribution of induction time. 15 experimental data points at a constant supersaturation were used to investigate the percentage of sample crystallised at different induction time. With the presence of 3D nanotemplate, the probability distribution of the induction time showed an exponential increase with time as shown in Fig. 2. It was observed that no significant increase in the percentage of crystallisation after 84 hours. However, IgG4 crystal was not formed without using $3 \mathrm{D}$ nanotemplate in the crystallisation process.

\section{CONCLUSION}

Conclusively, this paper presents the first report on 3D nanotemplate to facilitate the Crystallisation of IgG4. Without the presence of $3 \mathrm{D}$ nanotemplate, the crystallisation was found to be unsuccessful, producing no crystal at all at the same condition used in the successful IgG4 crystallisation. The identification of the optimum conditions of IgG4 crystallisation was achieved using the hanging drop vapour diffusion crystallisation techniques (with $750 \mu$ l reservoir solution and $6 \mu 1$ protein droplet). In the presence of $10 \mathrm{~nm}$ porosity 3D nanotemplates, the $\mathrm{IgG} 4$ was found to crystallise at a protein concentration of $10 \mathrm{mg} / \mathrm{ml}$, incubated in the environment with temperature of $20^{\circ} \mathrm{C}$ and neutral $\mathrm{pH}$, which consisting buffer solution (1M Imidazole and 0.2M Calcium Acetate) and precipitant solution (8\% w/v PEG 8000 as precipitant). The induction time reported by the author (6 hours) is much lower compared to that of the reported induction time by Smejkal et al. (2013), which is as long as a few weeks [15].

\section{REFERENCES}

[1] D. S. Tsekova, D. R. Williams, and J. Y. Y. Heng, "Effect of surface chemistry of novel templates on crystallization of proteins," Chemical Engineering Science, vol. 77, pp. 201-206, 2012.

[2] B. Ruiz, B. J. Coe, R. Gianotti, M. Jazbinsek, and P. Gunter, "Polymorphism, crystal growth and characterization of an organic nonlinear optical material: DAPSH," CrystEngComm, vol. 9, pp. 772-776, 2007.

[3] F. C. Meldrum and H. Cölfen, "Controlling mineral morphologies and structures in biological and synthetic systems," Chemical Reviews, vol. 108, pp. 4332-4432, 2008.

[4] J. D. Atkinson et al., "The importance of feldspar for ice nucleation by mineral dust in mixed-phase clouds," Nature, vol. 498, pp. 355-358, 2013.

[5] R. W. Hartel, "Advances in food crystallization," Annual Review of Food Science and Technology, vol. 4, pp. 277-292, 2013

[6] A. J. Smith et al., "Crystal engineering of green tea epigallocatechin-3-gallate (EGCg) cocrystals and pharmacokinetic modulation in rats," Mol. Pharmaceutics, vol. 10, pp. 2948-2961, 2013.

[7] Y. W, Wang, H. K. Christenson, and F. C. Meldrum, "Confinement leads to control over calcium sulfate polymorph," Advanced Functional Materials, vol. 23, pp. 5615-5623, 2013.

[8] Y. Diao, L. Shaw, Z. Bao, and S. C. B. Mannsfeld, "Morphology control strategies for solution-processed organic semiconductor thin films," Energy \& Environmental Science, vol. 7, pp. 2145-2159, 2014

[9] Y. Diao, A. S. Myerson, T. A. Hatton, and B. L. Trout, "Surface design for controlled crystallization: The role of surface chemistry and nanoscale pores in heterogeneous nucleation," Langmuir, vol. 27, pp. 5324-5334, 2011.

[10] X. Y. Liu, "A new kinetic model for three-dimensional heterogeneous nucleation," The Journal of Chemical Physics, vol. 111, pp. 1628-1630, 1999.

[11] A. Y. Lee, I. S. Lee, S. S. Dette, J. Boerner, and A. S. Myerson, "Crystallization on confined engineered surfaces: A method to control crystal size and generate different polymorphs," Journal of the American Chemical Society, vol. 127, pp. 14982-14983, 2005.

[12] L. Tan, R. M. Davis, A. S. Myerson, and B. L. Trout, "Control of heterogeneous nucleation via rationally designed biocompatible 
polymer surfaces with nanoscale features," Crystal Growth and Design, vol. 15, pp. 2176-2186, 2015.

[13] J. Urbanus et al., "Template Induced Crystallization: A relation between template properties and template performance," Crystal Growth and Design, vol. 9, pp. 2762-2769, 2009.

[14] U. V. Shah, D. R. Williams, and J. Y. Y. Heng, "Selective crystallization of proteins using engineered nanonucleantsselective crystallization of proteins using engineered nanonucleants," Crystal Growth and Design, vol. 12, pp. 1362-1369, 2012.

[15] B. Smejkal et al., "Fast and scalable purification of a therapeutic full-length antibody based on process crystallization," Biotechnology and Bioengineering, vol. 110, pp. 2452-2461, 2013.

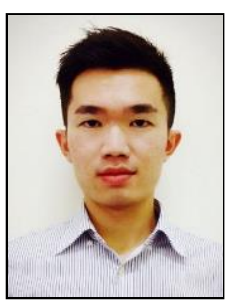

Kuan L. Tan was born on March 1, 1992 at Port Dickson, Malaysia. He is now an undergraduate student of chemical engineering from Faculty of Engineering Technology and Build Environment (FETBE), UCSI University, Cheras Malaysia. He is expected to receive his BEng (Hons) Chemical Engineering in August 2016. In 2015, he was involved in the Summer Research Program, conducted an experimental research on protein crystallization at Imperial College.

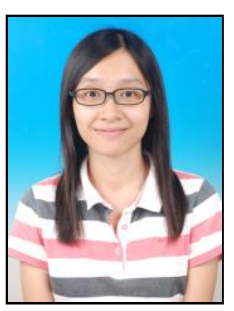

Peck L. Kiew accomplished her Ph.D. in biochemica engineering from Universiti Sains Malaysia (USM) in 2014 after obtaining bachelor degree in engineering (chemical engineering) at USM, Engineering Campus, Penang in October 2010. Her research work basically revolves within the areas of food and pharmaceutical products. In the past, she had been working on the extraction of type I collagen from Malaysian cultured freshwater fishes, especially from the hybrid catfish of Clarias gariepinus x C. microcephalus. Through the results, the possibility to convert this natural resource into value-added products has been proven. Thus it marks the possibility to improve its commercial value whilst assisting in ensuring the sustainability of Malaysian freshwater fishes. Work on optimization and kinetic or modeling studies are also undertaken in parallel to make sure that the optimum extraction process can be achieved. In short, her research interest encompasses on the exploitation of natural resources or waste materials that are abundant in Malaysia and converting them into value added products.

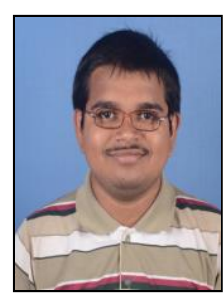

Nilanjon Naskar earned his bachelor of technology and master of technology in chemical engineering at Indian Institute of Technology Kharagpur in July, 2015. He received the Imperial College Visiting Student Scholarship 2015. During his stay at Imperial College London, he carried out research work on IgC crystallization under the supervision of Dr. Jerry Y.Y. Heng for the period: May-July, 2015.

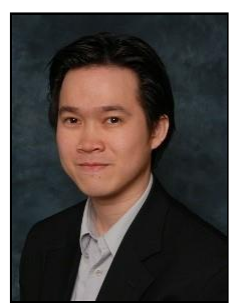

Jerry Y. Y. Heng received his bachelor degree in the Department of Chemical Engineering, Universiti Teknologi Malaysia, Malaysia in 2002. In 2006, he received his Ph.D. degree in the field of anisotropic surface properties of crystalline pharmaceutical solids from Department of Chemical Engineering, Imperial College London, United Kingdom. He is now a senior lecturer in the Department of Chemical Engineering, Imperial College London, United Kingdom since 2011. His principal area of research is to develop an improved fundamental understanding of the role of surface properties in particle engineering. The research activities he actively involved include studying the impact of particle engineering unit operations on surface properties, investigate the effects of particle properties on unit operations (eg granuation, drying), employ modeling tools for the predictions and control of these operations, etc. Dr. Jerry Y. Y. Heng is leading the Surfaces and Particle Engineering Laboratory (SPEL), which is one of the research groups within the Department of Chemical Engineering Imperial College London In 2013, he received a Royal Academy of Engineering ExxonMobil Teaching Excellence Award. Besides that, he is a member of IChemE and AAPS, a committee member of the Characterisation of Particulate Solids Working Party (CHoPS), European Federation of Chemical Engineers (EFCE), 2013 and also the treasurer for Particle Technology Subject Interest Group, Institution of Chemical Engineers, United Kingdom, 2011. 